\title{
Spiral morphology in an intensely star-forming disk galaxy more than 12 billion years ago
}

\author{
Takafumi Tsukui ${ }^{1,2 *}$ and Satoru Iguchi ${ }^{1,2}$ \\ ${ }^{1}$ Department of Astronomical Science, SOKENDAI (The Graduate University for Advanced Studies), 2-21-1 Osawa, Mitaka, Tokyo, Japan. ${ }^{2}$ National Astronomical Observatory \\ of Japan, National Institute of Natural Sciences, 2-21-1 Osawa, Mitaka, Tokyo, Japan. \\ ${ }^{*}$ Corresponding author. Email: t.tsukui@grad.nao.ac.jp
}

Spiral galaxies have distinct internal structures including a stellar bulge, disk and spiral arms. It is unknown when in cosmic history these structures formed. We analyze observations of BRI 1335-0417, an intensely star-forming galaxy in the distant Universe, at redshift 4.41. The [C II] gas kinematics show a steep velocity rise near the galaxy center and have a two-armed spiral morphology, which extends from about 2 to 5 kiloparsecs in radius. We interpret these features as due to a central compact structure, such as a bulge; a rotating gas disk; and either spiral arms or tidal tails. These features had formed within 1.4 billion years after the Big Bang, long before the peak of cosmic star formation.

Spiral galaxies contain distinct internal structures: a stellar bulge; a flat, extended rotating disk; and spiral arms that extend through the disk. Observations have established that the total star formation rate in the Universe rose steeply after the Big Bang, reached a peak at redshift $z$ between 1.5 and 3, and declined steadily since then (1). Optical observations provide evidence that stellar bulges have already formed in some massive galaxies by $z \sim 0.5$ to 2.5 (2). Spiral structure has been reported in galaxies at redshifts of $z=2.18(3), z=$ 2.01 (4) and $z=2.54$ (5). Observations with the Atacama Large Millimeter/submillimeter Array (ALMA) have shown spiral structure in two galaxies at $z=2.32$ and $z=2.67(6)$, dynamically cold disks in galaxies at redshift $z \sim 4(7-10)$ and compact central structures, like a bulge, at $z \sim 4$ to $5(7,10)$. It is currently unclear whether the later appearance of spiral structure is due to different formation times or observational limitations.

BRI 1335-0417 (QSO J1338-0432) is a galaxy at redshift z $=4.4074 \pm 0.0015$ (11), corresponding to $1.4 \mathrm{Gyr}$ after the Big Bang. It is classified as a Hyper Luminous InfraRed Galaxy (HyLIRG) with a luminosity of $3.1 \times 10^{13} L_{\odot}$ (where $L_{\odot}$ is the luminosity of the Sun) at far-infrared wavelengths (12) and a high star formation rate of $5 \pm 1 \times 10^{3} M_{\odot} \mathrm{yr}^{-1}$ (where $M_{\odot}$ is the mass of the Sun), estimated from modeling the spectral energy distribution (13).

We analyzed archival ALMA observations of BRI 13350417 in the gas emission line [C II] (rest frame frequency of $1900.5369 \mathrm{GHz}$, redshifted to around $351 \mathrm{GHz}$ in the observed frame). The data were reduced using standard procedures (14). [C II] line emission from this galaxy was detected in previous observations at lower spatial resolution, which was insufficient to determine the gas distribution and kinematics (15). Figure 1, A and B, shows the ALMA [C II] intensity and velocity images, with a spatial resolution of $0.20^{\prime \prime} \times 0.16^{\prime \prime}$ full width half maximum (FWHM), which is equivalent to $\sim 1.33 \times 1.11$ kiloparsecs $(\mathrm{kpc})$ at this redshift. Figure 1C shows the dust continuum image at a central frequency of $1863 \mathrm{GHz}$ (rest frame) with spatial resolution of $0.17^{\prime \prime} \times 0.14^{\prime \prime}(\sim 1.16 \times 0.95 \mathrm{kpc})$. The root mean square noise in each velocity channel (width of $20 \mathrm{~km} \mathrm{~s}^{-1}$ ) is about 0.35 millijansky per beam (mJy beam $\left.{ }^{-1}\right)$, while that of the dust continuum map is about $0.036 \mathrm{mJy}^{\text {beam }}{ }^{-1}$. Figure 1D shows a composite image of the gas velocities, produced from [C II] velocity channel maps (Fig. 2) that show the [C II] line intensity images at each velocity. In each figure, right ascension (RA) and declination (Dec) offsets are given relative to the position RA $=13^{\mathrm{h}} 38^{\mathrm{m}} 03^{\mathrm{s}} .416$, Dec $=-04^{\mathrm{o}} 32^{\prime} 35^{\prime \prime} .02(\mathrm{~J} 2000$ equinox).

The [C II] line and dust continuum images show a central compact structure surrounded by more extended emission, which could indicate a disk. A spiral morphology is apparent in Fig. 1D, with northern and southern arms extending from 2 to $5 \mathrm{kpc}$ in radius. This spiral morphology is more apparent in the [C II] velocity channel maps (Fig. 2). We compare these maps to previously published CO images (in the $J=2 \rightarrow 1$ line, where $J$ is the rotational quantum number) which showed different spatial distribution depending on the antenna configuration used for the observations. One CO image showed a structure with a bright central component and a weaker northern component (16), while another showed a complex structure with multiple components (17). Those multiple CO components correspond (in position and velocity) to the northern spiral feature, southern spiral feature and the central disk visible in our [C II] images. [C II] line emission arises from multiple phases of the interstellar medium, including 
ionized gas, atomic gas and molecular gas, while CO $(J=$ $2 \rightarrow 1$ ) line emission traces only dense molecular gas (18). The difference between [C II] and $\mathrm{CO}$ distributions also results from the differences in sensitivity and spatial frequency coverage between the observations. We found no evidence in the [C II] line emission of a bipolar outflow that has been reported based on $\mathrm{CO}(J=7 \rightarrow 6)$ observations (14).

The [C II] line velocity rises steeply in the center of the galaxy, reaching about $240-260 \mathrm{~km} \mathrm{~s}^{-1}$, indicating the presence of a centrally concentrated mass distribution. The velocity is approximately constant at $70-110 \mathrm{~km} \mathrm{~s}^{-1}$ in the outer parts of the galaxy, along the kinematic major axis, which we show in a position-velocity diagram (PVD; Fig. 1E). A steeply rising and/or constant velocity curve has been measured in other high redshift galaxies $(7,8,10)$. Such a velocity curve has been widely observed in nearby spiral galaxies, where it is produced by the gravitational potential of the internal structures (19). The [C II] PVD of BRI 1335-0417 indicates the presence of a central compact structure, such as a stellar bulge, and an extended disk. The rise in velocity appears to be steeper on the northern side than the southern side. A decrease in velocity occurs within a radius of $0.3^{\prime \prime}$ on the southern side, while a slight increase with radius is seen in the northern side. These features could be due to streaming motions along spiral arms, warping of the disk, recent gas accretion (10) and/or a lopsided central gas distribution. These disturbed features are common in nearby galaxies, and have also been found in high-redshift galaxies at $z \sim 4(8,10)$. Figure $1 \mathrm{~F}$ shows the velocity dispersions extracted at each position along the kinematic major axis. The velocity dispersions in the outer parts are consistent with a constant $\sim 70 \mathrm{~km} \mathrm{~s}^{-1}$, but increase up to $\sim 140 \mathrm{~km} \mathrm{~s}^{-1}$ in the center. Such central increases are known to be affected by the beam smearing effect (20) - because the spatial resolution is not sufficient to resolve the intrinsic velocity gradient, it is averaged over the beam width, so the observed velocity dispersion increases. We estimate an intrinsic velocity dispersion $\sigma$ of $71_{-11}^{+14} \mathrm{~km} \mathrm{~s}^{-1}$ (Fig. 1F) using a method (21) that extracts the velocity dispersions at the outer region of the disk, where the beam smearing is less severe.

We used Fourier analysis (3) to decompose the [C II] intensity image (Fig. 1A) into logarithmic spirals with $m$ arms (Fig. 3A) (14). The best-fitting model is a two-armed spiral morphology with a pitch angle of $26.7_{-1.6}^{+4.1 \circ}$ (95\% confidence level). Figure 3B shows this model overlain on the [C II] line intensity image after correction for the inclination angle of $37.8^{\circ}$ (i.e., de-projected to be viewed as face-on). The disk inclination was estimated from the axis ratio of the dust continuum image (table S1) (14). The amplitude of the $m=2$ signal is 6.0 times the noise, while the other modes $m=1,3,4$ have signal-to-noise ratios of 4.0, 2.5 and 2.4 respectively (Fig. 3A).
The $m=1$ mode corresponds to a one-arm spiral or lopsided morphology. The broad signal in $m=1$ mode (Fig. 3A) is due to the difference in length of two arms (Fig. 3B). The $m=3$ and 4 modes correspond to a triangular and boxy shape respectively, which could be produced by a stellar bar structure.

We estimate the inclination-corrected rotational velocity $v_{\text {rot }}$ is $179_{-18}^{+25} \mathrm{~km} \mathrm{~s}^{-1}$ at radius of $0.3^{\prime \prime}$, calculated using the flat part of the PVD (Fig. 1E). The ratio of $v_{\text {rot }}$ to the intrinsic velocity dispersion $\sigma$ quantifies the rotational support of the disk (20). Our estimated ratio $v_{\text {rot }} / \sigma=2.5_{-0.4}^{+0.6}$, indicating that the [C II] gas disk of this galaxy is rotation-dominated. Similar values have been found for other galaxies observed at $z$ $4(8,9)$. Higher ratios have also been reported at redshift of 4 to $5(7,10)$. Additional evidence of a rotating disk is a velocity field that is symmetric across the kinematic minor axis, as we observe in this object (Fig. 1B). Ordered rotation has been found in kinematic data of several galaxies at redshift $\sim 4$ (22), with such symmetric velocity fields.

We estimate the mass distribution by modeling gas motions under a gravitational potential, composed of a central compact structure and an extended rotating disk (14). The best-fitting effective radius of the compact structure $R_{\mathrm{e}}$ is less than $1.3 \mathrm{kpc}$ (at 95\% confidence interval); its mass is $5.2 \times 10^{9}$ to $3.0 \times 10^{10} M_{\odot}$ depending on the value of $R_{\text {e. }}$. These results are compatible with the typical effective radius and mass of stellar bulges in nearby galaxies (23). The modeled mass of the disk $4.9_{-2.5}^{+1.7} \times 10^{10} M_{\odot}$ is consistent with the molecular gas mass of $5.1 \times 10^{10} M_{\odot}$ (16) estimated from the CO $(J=2 \rightarrow 1)$ luminosity (assuming BRI 1335-0417 has element abundances equal to the Sun). We computed the gas disk Toomre parameter $Q$, which quantifies the gravitational stability of the disk against perturbations (14). We find $Q$ is less than 1 throughout the outer part of the disk (fig. S6), indicating the gas disk is susceptible to gravitational collapse, star formation (7-9) and spontaneous formation of spiral structure $(3,24)$.

Studies of nearby galaxies have shown that bulges and supermassive black holes coexist in massive galaxies, and that there is a strong correlation between their properties (25). The mass of the central black hole in BRI 1335-0417 has previously been estimated as $M_{\mathrm{BH}} \sim 6 \times 10^{9} M_{\odot}(26)$, although there are systematic effects that could bias this value (27). Our modeled compact structure mass $5.2 \times 10^{9}-3.0 \times 10^{10} M_{\odot}$ is consistent with the estimated $M_{\mathrm{BH}}$. The ratio of the black hole mass to the compact structure mass is 1 to 0.2 , which is higher than the black hole to bulge mass ratio of 0.001 to 0.002 observed in nearby galaxies (25) and includes the possibility of a supermassive black hole without a surrounding 
bulge. There is tentative evidence that the black hole-bulge mass ratio in high redshift galaxies could be higher than at low redshift, but the data are inconclusive (28).

Dusty starburst galaxies like BRI 1335-0417 are the progenitors of present-day massive elliptical galaxies, which are dominated by old stellar populations (7). This transformation requires contraction of the disk by angular momentum transport, through gravitational torques driven by nonaxisymmetric structures (29). The spiral morphology in BRI 1335-0417 is present at $z=4.41$, long before the peak of cosmic star formation. Spiral structure could influence the evolution of this galaxy: the angular momentum could be redistributed, triggering gas inflow into the center of the galaxy and driving intense star formation there (29). Such gas inflow cannot be directly detected in our data due to the limited signal to noise ratio.

The two-armed spiral morphology observed in BRI 13350417 could have been induced in the disk through tidal interactions $(3,24)$. Because this galaxy has a rotating disk and a compact structure at its center, sufficient time for their formation is likely to have passed after any major merger event. Cosmological simulations at redshift of $6(30)$ show that spiral structure in a rotating disk can appear when the disk is relaxed after a merger event. The two-armed spiral morphology in BRI 1335-0417 extends out to $5 \mathrm{kpc}$ (Fig. 1A), is connected to the galaxy center (Fig. 1D) and is associated with the rotating disk spatially and in velocity (Fig. 2 and Fig. 1E). Tidal-tail features in a galaxy merger are expected occur on larger scales $(>10 \mathrm{kpc})(6,31)$. We therefore disfavor, but cannot completely rule out, a tidal-tail origin for the observed spiral morphology. Alternatively, a stellar bar structure could play a role in forming the two-armed spiral structure $(24,32)$. Figure 1, A and C, shows non-axisymmetric structures, which could be due to a stellar bar, and the spiral arms appear to begin from the edge of this bar structure, as seen in nearby disk galaxies and numerical simulation (32).

The high star formation rates of $z>4$ galaxies like BRI 1335-0417 are commonly explained as the result of major mergers, which could produce distorted galactic kinematics. We find that BRI 1335-0417 has only slightly disturbed, rotation dominated kinematics, which can be well described by a rotating disk model (14). This suggests that the high star formation rate must have been maintained long enough for the disk to form after any major merger event. The $Q$ parameter shows the outer disk of BRI 1335-0417 is unstable, which could be caused by gas accretion along large-scale filaments of the cosmic web $(29,33)$, and/or minor mergers with accreting satellites (30).

\section{REFERENCES AND NOTES}

1. P. Madau, M. Dickinson, Cosmic Star-Formation History. Annu. Rev. Astron. Astrophys. 52, 415-486 (2014). doi:10.1146/annurev-astro-081811-125615

2. S. Tacchella, C. M. Carollo, A. Renzini, N. M. Förster Schreiber, P. Lang, S. Wuyts, G. Cresci, A. Dekel, R. Genzel, S. J. Lilly, C. Mancini, S. Newman, M. Onodera, A.
Shapley, L. Tacconi, J. Woo, G. Zamorani, Evidence for mature bulges and an inside-out quenching phase 3 billion years after the Big Bang. Science 348, 314317 (2015). doi:10.1126/science.1261094 Medline

3. D. R. Law, A. E. Shapley, C. C. Steidel, N. A. Reddy, C. R. Christensen, D. K. Erb, High velocity dispersion in a rare grand-design spiral galaxy at redshift $z=2.18$. Nature 487, 338-340 (2012). doi:10.1038/nature11256 Medline

4. S. Dawson, N. McCrady, D. Stern, M. E. Eckart, H. Spinrad, M. C. Liu, J. R. Graham, Optical and Near-Infrared Spectroscopy of a High-Redshift Hard X-Ray-emitting Spiral Galaxy. Astron. J. 125, 1236-1246 (2003). doi:10.1086/367792

5. T. Yuan, J. Richard, A. Gupta, C. Federrath, S. Sharma, B. A. Groves, L. J. Kewley, R. Cen, Y. Birnboim, D. B. Fisher, The Most Ancient Spiral Galaxy: A 2.6-Gyr-old Disk with a Tranquil Velocity Field. Astrophys. J. 850, 61 (2017). doi:10.3847/15384357/aa951d

6. J. A. Hodge, I. Smail, F. Walter, E. Cunha, A. M. Swinbank, M. Rybak, B. Venemans, W. N. Brandt, G. C. Rivera, S. C. Chapman, C.-C. Chen, P. Cox, H. Dannerbauer, R. Decarli, T. R. Greve, K. K. Knudsen, K. M. Menten, E. Schinnerer, J. M. Simpson, P. Werf, J. L. Wardlow, A. Weiss, ALMA Reveals Potential Evidence for Spiral Arms, Bars, and Rings in High-redshift Submillimeter Galaxies. Astrophys. J. 876, 130 (2019). doi:10.3847/1538-4357/ab1846

7. F. Rizzo, S. Vegetti, D. Powell, F. Fraternali, J. P. McKean, H. R. Stacey, S. D. M. White, A dynamically cold disk galaxy in the early Universe. Nature 584, 201-204 (2020). doi:10.1038/s41586-020-2572-6 Medline

8. M. Neeleman, J. X. Prochaska, N. Kanekar, M. Rafelski, A cold, massive, rotating disk galaxy 1.5 billion years after the Big Bang. Nature 581, 269-272 (2020). doi:10.1038/s41586-020-2276-y Medline

9. K. Tadaki, D. Iono, M. S. Yun, I. Aretxaga, B. Hatsukade, D. H. Hughes, S. Ikarashi, T. Izumi, R. Kawabe, K. Kohno, M. Lee, Y. Matsuda, K. Nakanishi, T. Saito, Y. Tamura, J. Ueda, H. Umehata, G. W. Wilson, T. Michiyama, M. Ando, P. Kamieneski, The gravitationally unstable gas disk of a starburst galaxy 12 billion years ago. Nature 560, 613-616 (2018). doi:10.1038/s41586-018-0443-1 Medline

10. F. Lelli, E. M. Di Teodoro, F. Fraternali, A. W. S. Man, Z.-Y. Zhang, C. De Breuck, T. A. Davis, R. Maiolino, A massive stellar bulge in a regularly rotating galaxy 1.2 billion years after the Big Bang. Science 371, 713-716 (2021). doi:10.1126/science.abc1893 Medline

11. S. Guilloteau, A. Omont, R. G. McMahon, P. Cox, P. Petitjean, The second detection of CO at redshift larger than 4. Astron. Astrophys. 328, L1 (1997).

12. C. L. Carilli, K. Kohno, R. Kawabe, K. Ohta, C. Henkel, K. M. Menten, M. S. Yun, A. Petric, Y. Tutui, High-Resolution Imaging of Molecular Line Emission from HighRedshift QSOs. Astron. J. 123, 1838-1846 (2002). doi:10.1086/339306

13. J. Wagg, C. L. Carilli, M. Aravena, P. Cox, L. Lentati, R. Maiolino, R. G. McMahon, D. Riechers, F. Walter, P. Andreani, R. Hills, A. Wolfe, Karl G. Jansky Very Large Array Observations of Cold Dust and Molecular Gas in Starbursting Quasar Host Galaxies at z 4.5. Astrophys. J. 783, 71 (2014). doi:10.1088/0004$637 \times / 783 / 2 / 71$

14. Materials and methods are available as supplementary materials.

15. J. Wagg, C. L. Carilli, D. J. Wilner, P. Cox, C. De Breuck, K. Menten, D. A. Riechers, F. Walter, [CII] line emission in BRI 1335-0417 at z = 4.4. Astron. Astrophys. 519, L1 (2010). doi:10.1051/0004-6361/201015424

16. G. C. Jones, C. L. Carilli, E. Momjian, J. Wagg, D. A. Riechers, F. Walter, R. Decarli, K. Ota, R. McMahon, New constraints on the molecular gas in the prototypical HyLIRGs BRI 1202-0725 and BRI 1335-0417. Astrophys. J. 830, 63 (2016). doi:10.3847/0004-637x/830/2/63

17. D. A. Riechers, F. Walter, C. L. Carilli, F. Bertoldi, E. Momjian, Formation of a Quasar Host Galaxy through a Wet Merger 1.4 Billion Years after the Big Bang. Astrophys. J. 686, L9-L12 (2008). doi:10.1086/592834

18. J. L. Pineda, W. D. Langer, T. Velusamy, P. F. Goldsmith, A Herschel [C II] Galactic plane survey. I. The global distribution of ISM gas components. Astron. Astrophys. 554, A103 (2013). doi:10.1051/0004-6361/201321188

19. Y. Sofue, J. Koda, H. Nakanishi, S. Onodera, The Virgo High-Resolution CO Survey: II. Rotation Curves and Dynamical Mass Distributions. Publ. Astron. Soc. Jpn. 55, 59-74 (2003). doi:10.1093/pasj/55.1.59

20. A. Burkert, N. M. F. Schreiber, R. Genzel, P. Lang, L. J. Tacconi, E. Wisnioski, S. Wuyts, K. Bandara, A. Beifiori, R. Bender, G. Brammer, J. Chan, R. Davies, A. Dekel, M. Fabricius, M. Fossati, S. Kulkarni, D. Lutz, J. T. Mendel, I. Momcheva, E. J. Nelson, T. Naab, A. Renzini, R. Saglia, R. M. Sharples, A. Sternberg, D. Wilman, E. 
Wuyts, The Angular Momentum Distribution and Baryon Content of Star-forming Galaxies at $z \sim 1-3$. Astrophys. J. 826, 214 (2016). doi:10.3847/0004$637 X / 826 / 2 / 214$

21. A. W. Green, K. Glazebrook, P. J. McGregor, R. G. Abraham, G. B. Poole, I. Damjanov, P. J. McCarthy, M. Colless, R. G. Sharp, High star formation rates as the origin of turbulence in early and modern disk galaxies. Nature 467, 684-686 (2010). doi:10.1038/nature09452 Medline

22. G. C. Jones, C. L. Carilli, Y. Shao, R. Wang, P. L. Capak, R. Pavesi, D. A. Riechers, A. Karim, M. Neeleman, F. Walter, Dynamical Characterization of Galaxies at z 4-6 via Tilted Ring Fitting to ALMA [C II] Observations. Astrophys. J. 850, 180 (2017). doi:10.3847/1538-4357/aa8df2

23. D. A. Gadotti, Structural properties of pseudo-bulges, classical bulges and elliptical galaxies: A Sloan Digital Sky Survey perspective. Mon. Not. R. Astron. Soc. 393, 1531-1552 (2009). doi:10.1111/i.1365-2966.2008.14257x

24. C. Dobbs, J. Baba, Dawes Review 4: Spiral Structures in Disc Galaxies. Publ. Astron. Soc. Aust. 31, e035 (2014). doi:10.1017/pasa.2014.31

25. J. Kormendy, L. C. Ho, Coevolution (Or Not) of Supermassive Black Holes and Host Galaxies. Annu. Rev. Astron. Astrophys. 51, 511-653 (2013). doi:10.1146/annurevastro-082708-101811

26. G. A. Shields, K. L. Menezes, C. A. Massart, P. Vanden Bout, The Black Hole-Bulge Relationship for QSOs at High Redshift. Astrophys. J. 641, 683-688 (2006). doi:10.1086/500542

27. J. E. Mejía-Restrepo, P. Lira, H. Netzer, B. Trakhtenbrot, D. M. Capellupo, The effect of nuclear gas distribution on the mass determination of supermassive black holes. Nat. Astron. 2, 63-68 (2018). doi:10.1038/s41550-017-0305-z

28. A. Schulze, L. Wisotzki, Accounting for selection effects in the BH-bulge relations: No evidence for cosmological evolution. Mon. Not. R. Astron. Soc. 438, 34223433 (2014). doi:10.1093/mnras/stt2457

29. A. Dekel, A. Burkert, Wet disc contraction to galactic blue nuggets and quenching to red nuggets. Mon. Not. R. Astron. Soc. 438, 1870-1879 (2014). doi:10.1093/mnras/stt2331

30. M. Kohandel, A. Pallottini, A. Ferrara, A. Zanella, C. Behrens, S. Carniani, S. Gallerani, L. Vallini, Kinematics of $\mathrm{z} \geq 6$ galaxies from [C II] line emission. Mon. Not. R. Astron. Soc. 487, 3007-3020 (2019). doi:10.1093/mnras/stz1486

31. J. Ren, X. Z. Zheng, D. Valls-Gabaud, P.-A. Duc, E. F. Bell, Z. Pan, J. Qin, D. D. Shi, M. Qiao, Y. He, R. Wen, Long Tidal Tails in Merging Galaxies and Their Implications. Mon. Not. R. Astron. Soc. 499, 3399-3409 (2020). doi:10.1093/mnras/staa2985

32. J. Baba, Short-term dynamical evolution of grand-design spirals in barred galaxies. Mon. Not. R. Astron. Soc. 454, 2954-2964 (2015). doi:10.1093/mnras/stv2220

33. A. Dekel, Y. Birnboim, G. Engel, J. Freundlich, T. Goerdt, M. Mumcuoglu, E. Neistein, C. Pichon, R. Teyssier, E. Zinger, Cold streams in early massive hot haloes as the main mode of galaxy formation. Nature 457, 451-454 (2009). doi:10.1038/nature07648 Medline

34. C. L. Bennett, D. Larson, J. L. Weiland, G. Hinshaw, The $1 \%$ Concordance Hubble Constant. Astrophys. J. 794, 135 (2014). doi:10.1088/0004-637X/794/2/135

35. S. Iguchi, Radio Interferometer Sensitivities for Three Types of Receiving Systems: DSB, SSB, and 2SB Systems. Publ. Astron. Soc. Jpn. 57, 643-677 (2005). doi:10.1093/pasj/57.4.643

36. A. Remijan, A. Biggs, P. Cortes, B. Dent, J. Di Francesco, E. Fomalont, A. Hales, S. Kameno, B. Mason, N. Philips, K. Saini, F. Stoehr, B. Vila Vilaro, E. Villard, ALMA Cycle 7 Technical Handbook (2019), doi:10.5281/zenodo.4511522.

37. J. P. McMullin, B. Waters, D. Schiebel, W. Young, K. Golap, in Astronomical Data Analysis Software and Systems XVI, R. A. Shaw, F. Hill, D. J. Bell, Eds., vol. 376 of Astronomical Society of the Pacific Conference Series (Astronomical Society of the Pacific, 2007), p. 127.

38. D. S. Briggs, High Fidelity Interferometric Imaging: Robust Weighting and NNLS Deconvolution. Bull. Am. Astron. Soc. 27, 1444 (1995).

39. T. M. Dame, Optimization of Moment Masking for CO Spectral Line Surveys. arXiv preprint (2011); https://arxiv.org/abs/1101.1499.

40. T. A. Davis, M. Bureau, M. Cappellari, M. Sarzi, L. Blitz, A black-hole mass measurement from molecular gas kinematics in NGC4526. Nature 494, 328-330 (2013). doi:10.1038/nature11819 Medline

41. D. Krajnović, M. Cappellari, P. T. De Zeeuw, Y. Copin, Kinemetry: A generalization of photometry to the higher moments of the line-of-sight velocity distribution. Mon. Not. R. Astron. Soc. 366, 787-802 (2006). do:10.1111/j,1365-2966.2005.09902.x
42. C. Y. Peng, L. C. Ho, C. D. Impey, H.-W. Rix, Detailed Decomposition of Galaxy Images. II. Beyond Axisymmetric Models. Astron. J. 139, 2097-2129 (2010). doi:10.1088/0004-6256/139/6/2097

43. R. Lange, A. J. Moffett, S. P. Driver, A. S. G. Robotham, C. P. Lagos, L. S. Kelvin, C. Conselice, B. Margalef-Bentabol, M. Alpaslan, I. Baldry, J. Bland-Hawthorn, M. Bremer, S. Brough, M. Cluver, M. Colless, L. J. M. Davies, B. Häußler, B. W. Holwerda, A. M. Hopkins, P. R. Kafle, R. Kennedy, J. Liske, S. Phillipps, C. C. Popescu, E. N. Taylor, R. Tuffs, E. van Kampen, A. H. Wright, Galaxy And Mass Assembly (GAMA): $M_{\star}-R_{\mathrm{e}}$ relations of $\mathrm{z}=0$ bulges, discs and spheroids. Mon. Not. R. Astron. Soc. 462, 1470-1500 (2016). doi:10.1093/mnras/stw1495

44. S. P. Driver, P. D. Allen, A. W. Graham, E. Cameron, J. Liske, S. C. Ellis, N. J. G. Cross, R. De Propris, S. Phillipps, W. J. Couch, The Millennium Galaxy Catalogue: Morphological classification and bimodality in the colour-concentration plane. Mon. Not. R. Astron. Soc. 368, 414-434 (2006). doi:10.1111/i.13652966.2006.10126.x

45. S. van der Walt, J. L. Schönberger, J. Nunez-Iglesias, F. Boulogne, J. D. Warner, N. Yager, E. Gouillart, T. Yu; scikit-image contributors, scikit-image: Image processing in Python. Peer J 2, e453 (2014). doi:10.7717/peeri.453 Medline

46. I. Puerari, H. A. Dottori, Fourier analysis of structure in spiral galaxies. Astron. Astrophys. Suppl. Ser. 93, 469 (1992).

47. M. D. Smith, M. Bureau, T. A. Davis, M. Cappellari, L. Liu, E. V. North, K. Onishi, S. Iguchi, M. Sarzi, WISDOM project - IV. A molecular gas dynamical measurement of the supermassive black hole mass in NGC 524. Mon. Not. R. Astron. Soc. 485, 4359-4374 (2019). doi:10.1093/mnras/stz625

48. S. M. Kent, Dark matter in spiral galaxies. I. Galaxies with optical rotation curves. Astron. J. 91, 1301 (1986). doi:10.1086/114106

49. Y. Sofue, M. Honma, T. Omodaka, Unified Rotation Curve of the GalaxyDecomposition into de Vaucouleurs Bulge, Disk, Dark Halo, and the 9-kpc Rotation Dip-. Publ. Astron. Soc. Jpn. 61, 227-236 (2009). doi:10.1093/pasj/61.2.227

50. R. Genzel, N. M. F. Schreiber, H. Übler, P. Lang, T. Naab, R. Bender, L. J. Tacconi, E. Wisnioski, S. Wuyts, T. Alexander, A. Beifiori, S. Belli, G. Brammer, A. Burkert, C. M. Carollo, J. Chan, R. Davies, M. Fossati, A. Galametz, S. Genel, O. Gerhard, D. Lutz, J. T. Mendel, I. Momcheva, E. J. Nelson, A. Renzini, R. Saglia, A. Sternberg, S. Tacchella, K. Tadaki, D. Wilman, Strongly baryon-dominated disk galaxies at the peak of galaxy formation ten billion years ago. Nature 543, 397-401 (2017). doi:10.1038/nature21685 Medline

51. N. M. Förster Schreiber, R. Genzel, N. Bouché, G. Cresci, R. Davies, P. Buschkamp, K. Shapiro, L. J. Tacconi, E. K. S. Hicks, S. Genel, A. E. Shapley, D. K. Erb, C. C. Steidel, D. Lutz, F. Eisenhauer, S. Gillessen, A. Sternberg, A. Renzini, A. Cimatti, E. Daddi, J. Kurk, S. Lilly, X. Kong, M. D. Lehnert, N. Nesvadba, A. Verma, H. McCracken, N. Arimoto, M. Mignoli, M. Onodera, The SINS Survey: SINFONI Integral Field Spectroscopy of $\mathrm{z} \sim 2$ Star-forming Galaxies. Astrophys. J. 706, 1364-1428 (2009). doi:10.1088/0004-637X/706/2/1364

52. S. Wuyts, N. M. F. Schreiber, E. Wisnioski, R. Genzel, A. Burkert, K. Bandara, A. Beifiori, S. Belli, R. Bender, G. B. Brammer, J. Chan, R. Davies, M. Fossati, A. Galametz, S. K. Kulkarni, P. Lang, D. Lutz, J. T. Mendel, I. G. Momcheva, T. Naab, E. J. Nelson, R. P. Saglia, S. Seitz, L. J. Tacconi, K. Tadaki, H. Übler, P. G. Dokkum, D. J. Wilman, E. Wuyts, KMOS3D: Dynamical Constraints on the Mass Budget in Early Star-forming Disks. Astrophys. J. 831, 149 (2016). doi:10.3847/0004$637 \times / 831 / 2 / 149$

53. P. Lang, S. Wuyts, R. S. Somerville, N. M. F. Schreiber, R. Genzel, E. F. Bell, G. Brammer, A. Dekel, S. M. Faber, H. C. Ferguson, N. A. Grogin, D. D. Kocevski, A. M. Koekemoer, D. Lutz, E. J. McGrath, I. Momcheva, E. J. Nelson, J. R. Primack, D. J. Rosario, R. E. Skelton, L. J. Tacconi, P. G. van Dokkum, K. E. Whitaker, Bulge Growth and Quenching since $z=2.5$ in CANDELS/3D-HST. Astrophys. J. 788, 11 (2014). doi:10.1088/0004-637X/788/1/11

54. V. A. Bruce, J. S. Dunlop, R. J. McLure, M. Cirasuolo, F. Buitrago, R. A. A. Bowler, T. A. Targett, E. F. Bell, D. H. McIntosh, A. Dekel, S. M. Faber, H. C. Ferguson, N. A. Grogin, W. Hartley, D. D. Kocevski, A. M. Koekemoer, D. C. Koo, E. J. McGrath, The bulge-disc decomposed evolution of massive galaxies at $1<z<3$ in CANDELS. Mon. Not. R. Astron. Soc. 444, 1001-1033 (2014). doi:10.1093/mnras/stu1478

55. G. de Vaucouleurs, Recherches sur les Nebuleuses Extragalactiques. Ann. Astrophys. 11, 247 (1948).

56. A. W. Graham, S. P. Driver, A Concise Reference to (Projected) Sérsic $R^{1 / n}$ Quantities, Including Concentration, Profile Slopes, Petrosian Indices, and Kron 
Magnitudes. Publ. Astron. Soc. Aust. 22, 118-127 (2005). doi:10.1071/AS05001

57. J. Binney, S. Tremaine, Galactic Dynamics: Second Edition (Princeton. Univ. Press, Princeton, NJ, 2008).

58. E. Noordermeer, The rotation curves of flattened Sérsic bulges. Mon. Not. R. Astron. Soc. 385, 1359-1364 (2008). doi:10.1111/i.1365-2966.2008.12837x

59. N. Katz, J. E. Gunn, Dissipational Galaxy Formation. I. Effects of Gasdynamics. Astrophys. J. 377, 365 (1991). doi:10.1086/170367

60. R. Genzel, N. M. Förster Schreiber, P. Lang, S. Tacchella, L. J. Tacconi, S. Wuyts, K. Bandara, A. Burkert, P. Buschkamp, C. M. Carollo, G. Cresci, R. Davies, F. Eisenhauer, E. K. S. Hicks, J. Kurk, S. J. Lilly, D. Lutz, C. Mancini, T. Naab, S. Newman, Y. Peng, A. Renzini, K. Shapiro Griffin, A. Sternberg, D. Vergani, E. Wisnioski, E. Wuyts, G. Zamorani, The SINS/zC-SINF Survey of z 2 Galaxy Kinematics: Evidence for Gravitational Quenching. Astrophys. J. 785, 75 (2014). doi:10.1088/0004-637X/785/1/75

61. A. Burkert, R. Genzel, N. Bouché, G. Cresci, S. Khochfar, J. Sommer-Larsen, A. Sternberg, T. Naab, N. F. Schreiber, L. Tacconi, K. Shapiro, E. Hicks, D. Lutz, R. Davies, P. Buschkamp, S. Genel, High-redshift Star-forming Galaxies: Angular Momentum and Baryon Fraction, Turbulent Pressure Effects, and the Origin of Turbulence. Astrophys. J. 725, 2324-2332 (2010). doi:10.1088/0004637X/725/2/2324

62. K. C. Freeman, On the Disks of Spiral and S0 Galaxies. Astrophys. J. 160, 811 (1970). doi:10.1086/150474

63. A. Zanella, E. Daddi, G. Magdis, T. Diaz Santos, D. Cormier, D. Liu, A. Cibinel, R. Gobat, M. Dickinson, M. Sargent, G. Popping, S. C. Madden, M. Bethermin, T. M. Hughes, F. Valentino, W. Rujopakarn, M. Pannella, F. Bournaud, F. Walter, T. Wang, D. Elbaz, R. T. Coogan, The [C II] emission as a molecular gas mass tracer in galaxies at low and high redshifts. Mon. Not. R. Astron. Soc. 481, 1976-1999 (2018). doi:10.1093/mnras/sty2394

64. B. Gullberg, A. M. Swinbank, I. Smail, A. D. Biggs, F. Bertoldi, C. D. Breuck, S. C. Chapman, C.-C. Chen, E. A. Cooke, K. E. K. Coppin, P. Cox, H. Dannerbauer, J. S. Dunlop, A. C. Edge, D. Farrah, J. E. Geach, T. R. Greve, J. Hodge, E. Ibar, R. J. Ivison, A. Karim, E. Schinnerer, D. Scott, J. M. Simpson, S. M. Stach, A. P. Thomson, P. van der Werf, F. Walter, J. L. Wardlow, A. Weiss, The Dust and [C II] Morphologies of Redshift $\sim 4.5$ Sub-millimeter Galaxies at $\sim 200$ pc Resolution: The Absence of Large Clumps in the Interstellar Medium at High-redshift. Astrophys. J. 859, 12 (2018). doi:10.3847/1538-4357/aabe8c

65. E. Wisnioski, N. M. Förster Schreiber, S. Wuyts, E. Wuyts, K. Bandara, D. Wilman, R. Genzel, R. Bender, R. Davies, M. Fossati, P. Lang, J. T. Mendel, A. Beifiori, G. Brammer, J. Chan, M. Fabricius, Y. Fudamoto, S. Kulkarni, J. Kurk, D. Lutz, E. J. Nelson, I. Momcheva, D. Rosario, R. Saglia, S. Seitz, L. J. Tacconi, P. G. van Dokkum, The KMOS ${ }^{3 D}$ Survey: Design, First Results, and the Evolution of Galaxy Kinematics from $0.7 \leq z \leq 2.7$. Astrophys. J. 799, 209 (2015). doi:10.1088/0004$\underline{637 X / 799 / 2 / 209}$

66. P. Lang, N. M. Förster Schreiber, R. Genzel, S. Wuyts, E. Wisnioski, A. Beifiori, S. Belli, R. Bender, G. Brammer, A. Burkert, J. Chan, R. Davies, M. Fossati, A. Galametz, S. K. Kulkarni, D. Lutz, J. T. Mendel, I. G. Momcheva, T. Naab, E. J. Nelson, R. P. Saglia, S. Seitz, S. Tacchella, L. J. Tacconi, K. Tadaki, H. Übler, P. G. van Dokkum, D. J. Wilman, Falling Outer Rotation Curves of Star-forming Galaxies at $0.6 \lesssim z \lesssim 2.6$ Probed with KMOS3D and SINS/ZC-SINF. Astrophys. J. 840, 92 (2017). doi:10.3847/1538-4357/aa6d82

67. D. Foreman-Mackey, D. W. Hogg, D. Lang, J. Goodman, emcee: The MCMC Hammer. Publ. Astron. Soc. Pac. 125, 306-312 (2013). doi:10.1086/670067

68. R. C. E. Van Den Bosch, G. Van De Ven, Recovering the intrinsic shape of early-type galaxies. Mon. Not. R. Astron. Soc. 398, 1117-1128 (2009). doi:10.1111/j.13652966.2009.15177x

69. E. V. North, T. A. Davis, M. Bureau, M. Cappellari, S. Iguchi, L. Liu, K. Onishi, M. Sarzi, M. D. Smith, T. G. Williams, WISDOM project - V. Resolving molecular gas in Keplerian rotation around the supermassive black hole in NGC 0383. Mon. Not. $R$. Astron. Soc. 490, 319-330 (2019). doi:10.1093/mnras/stz2598

70. M. Mitzkus, M. Cappellari, C. J. Walcher, Dominant dark matter and a counterrotating disc: MUSE view of the low-luminosity S0 galaxy NGC 5102. Mon. Not. $R$. Astron. Soc. 464, 4789-4806 (2017). doi:10.1093/mnras/stw2677

71. T. A. Davis, M. Bureau, K. Onishi, M. Cappellari, S. Iguchi, M. Sarzi, WISDOM Project - II. Molecular gas measurement of the supermassive black hole mass in NGC 4697. Mon. Not. R. Astron. Soc. 468, 4675-4690 (2017). doi:10.1093/mnras/stw3217
72. N. Lu, T. Cao, T. Díaz-Santos, Y. Zhao, G. C. Privon, C. Cheng, Y. Gao, C. K. Xu, V. Charmandaris, D. Rigopoulou, P. P. Werf, J. Huang, Z. Wang, A. S. Evans, D. B. Sanders, CO (7-6), [C I] $370 \mu \mathrm{m}$, and [N II] $205 \mu \mathrm{m}$ Line Emission of the QSO BRI 1335-0417 at Redshift 4.407. Astrophys. J. 864, 38 (2018). doi:10.3847/15384357/aad3c9

73. J. S. Spilker, K. A. Phadke, M. Aravena, M. Béthermin, S. C. Chapman, C. Dong, A. H. Gonzalez, C. C. Hayward, Y. D. Hezaveh, S. Jarugula, K. C. Litke, M. A. Malkan, D. P. Marrone, D. Narayanan, C. Reuter, J. D. Vieira, A. Weiss, Ubiquitous Molecular Outflows in z $>4$ Massive, Dusty Galaxies. I. Sample Overview and Clumpy Structure in Molecular Outflows on 500 pc Scales. Astrophys. J. 905, 85 (2020). doi:10.3847/1538-4357/abc47f

74. A. Toomre, On the gravitational stability of a disk of stars. Astrophys. J. 139, 12171238 (1964). doi:10.1086/147861

75. P. Goldreich, D. Lynden-Bell, I. Gravitational stability of uniformly rotating disks. Mon. Not. R. Astron. Soc. 130, 97-124 (1965). doi:10.1093/mnras/130.2.97

76. M. Cappellari, Improving the full spectrum fitting method: Accurate convolution with Gauss-Hermite functions. Mon. Not. R. Astron. Soc. 466, 798-811 (2017) doi:10.1093/mnras/stw3020

\section{ACKNOWLEDGMENTS}

We thank K. Tadaki, Y. Matsuda, Y. Harikane, D. Iono, I. Mitsuhashi and S. Fujimoto for discussions about high redshift submillimeter galaxies, K. Onishi and D. Nguyen for discussions on the gas dynamics in galaxies, and H. Mikami, J. Ueda and $\mathrm{H}$. Nagai for support of the ALMA data reduction. ALMA is a partnership of ESO (representing its member states), NSF (USA) and NINS (Japan), together with NRC (Canada), MOST and ASIAA (Taiwan), and KASI (South Korea), in cooperation with the Republic of Chile. The Joint ALMA Observatory is operated by ESO, AUI/NRAO and NAOJ. Data analysis was in part carried out on the common-use data analysis computer system at the East Asian ALMA Regional Center (EA ARC) and the Astronomy Data Center (ADC) of the National Astronomical Observatory of Japan (NAOJ). Funding: T.T was partially supported by Overseas Travel Fund for Students (2019) of the Department of Astronomical Science, the Graduate University for Advanced Studies, SOKENDAI. Author contributions: T.T. led the data analysis. T.T. and S.I. discussed the interpretation of the results and wrote the manuscript. Competing interests: We declare no competing interests. Data and materials availability: The ALMA raw and pipeline calibrated data are available at https://almascience.nrao.edu/aq/under project code 2017.1.00394.S. Some of the ALMA data were retrieved from the JVO portal http://jvo.nao.ac.jp/portal/ operated by ADC/NAOJ. The results of our analysis are available in supplementary data files: the observed [C II] data cube cutout used for dynamical modeling (data S1), the model [C II] data cube generated using KinMS (data S2), the observed full [C II] data cube used to extract the spectrum (data S3), and the observed dust continuum image (data S4). Our derived model values are listed in tables S1 and S2.

\section{SUPPLEMENTARY MATERIALS}

science.sciencemag.org/cgi/content/full/science.abe9680/DC1

Materials and Methods

Figs. S1 to S6

Tables S1 and S2

References (34-76)

Data S1 to S4

30 September 2020; accepted 30 April 2021

Published online 20 May 2021

10.1126/science.abe9680 

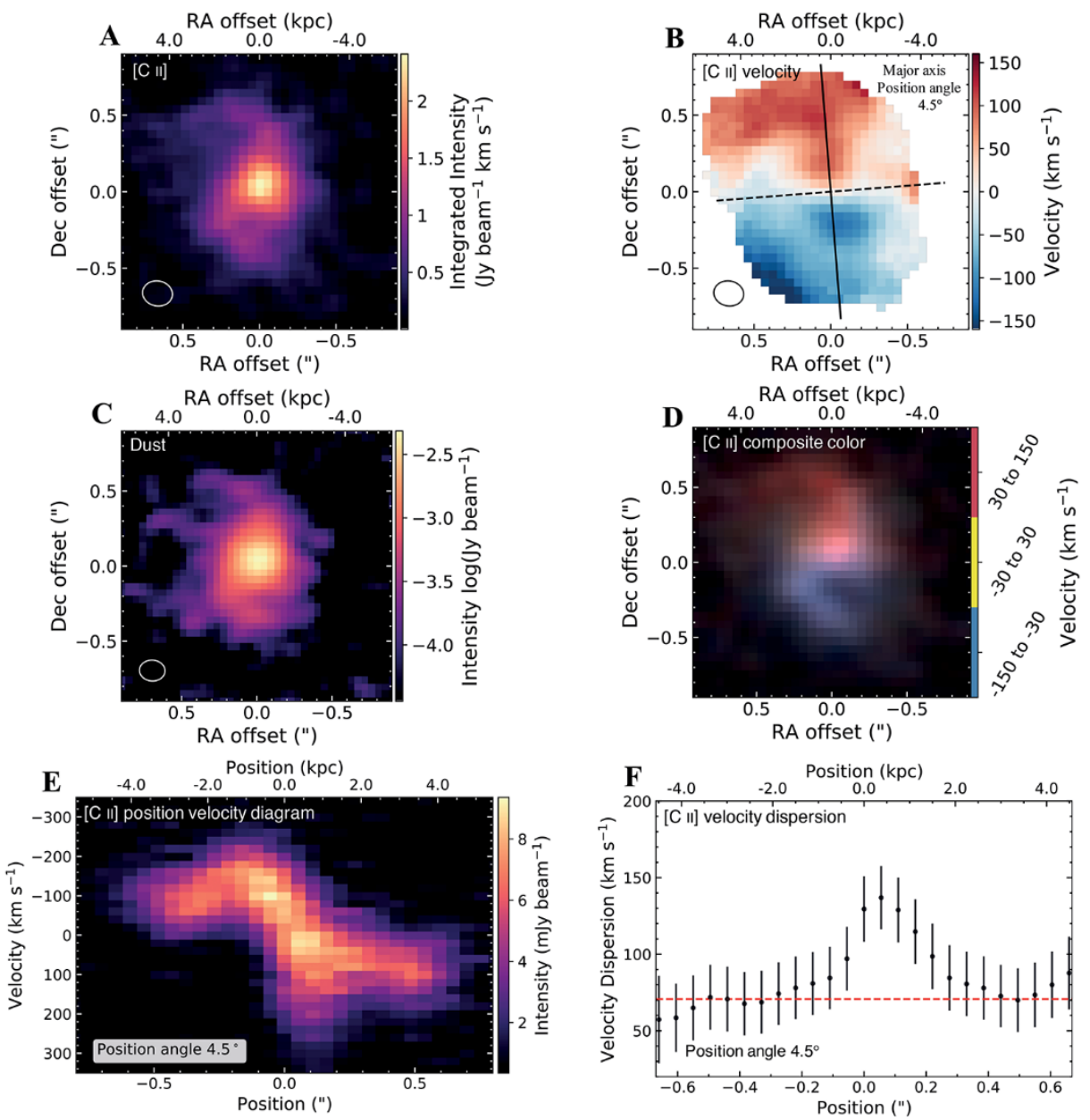

Fig. 1. Morphology and kinematics of [C II] line and dust continuum emission in BRI 1335-0417. (A) and (B) the ALMA [C II line maps of intensity (moment 0 ) and intensity-weighted velocity (moment 1 ). The kinematic major and minor axes are shown in panel B with black solid and dotted lines, respectively. (C) the ALMA dust continuum map at a restframe frequency of $1863 \mathrm{GHz}$. (D) a composite color image showing in red the redshifted gas emission with velocity of 30 to $150 \mathrm{~km} \mathrm{~s}^{-1}$, in yellow gas at the systemic velocity of -30 to $30 \mathrm{~km} \mathrm{~s}^{-1}$, and in blue the blueshifted gas with velocity of -150 to $-30 \mathrm{~km} \mathrm{~s}^{-1}$. The FWHM of the synthesized beam is $0.20^{\prime \prime} \times 0.16 "$ in (A), (B) and (D), and 0.17" $\times 0.14^{\prime \prime}$ in (C), which are shown with ellipses in the bottom left corners. (E) PVD of the observed [C I] line emission, extracted along the kinematic major axis shown in panel $B$, which has a position angle of $4.5^{\circ}$ (table S1). Data were averaging over one beam width (three pixels). (F) the velocity dispersion measured from the line profile at each position of the PVD. The error bars correspond to the sum in quadrature of the profile fitting uncertainty (95\% confidence level) and the velocity channel width. The red dashed line shows the estimated intrinsic velocity dispersion of $71_{-11}^{+14} \mathrm{~km} \mathrm{~s}^{-1}$. Positive position in panels $(E)$ and $(F)$ is the direction toward the north along the kinematic major axis in (B). 


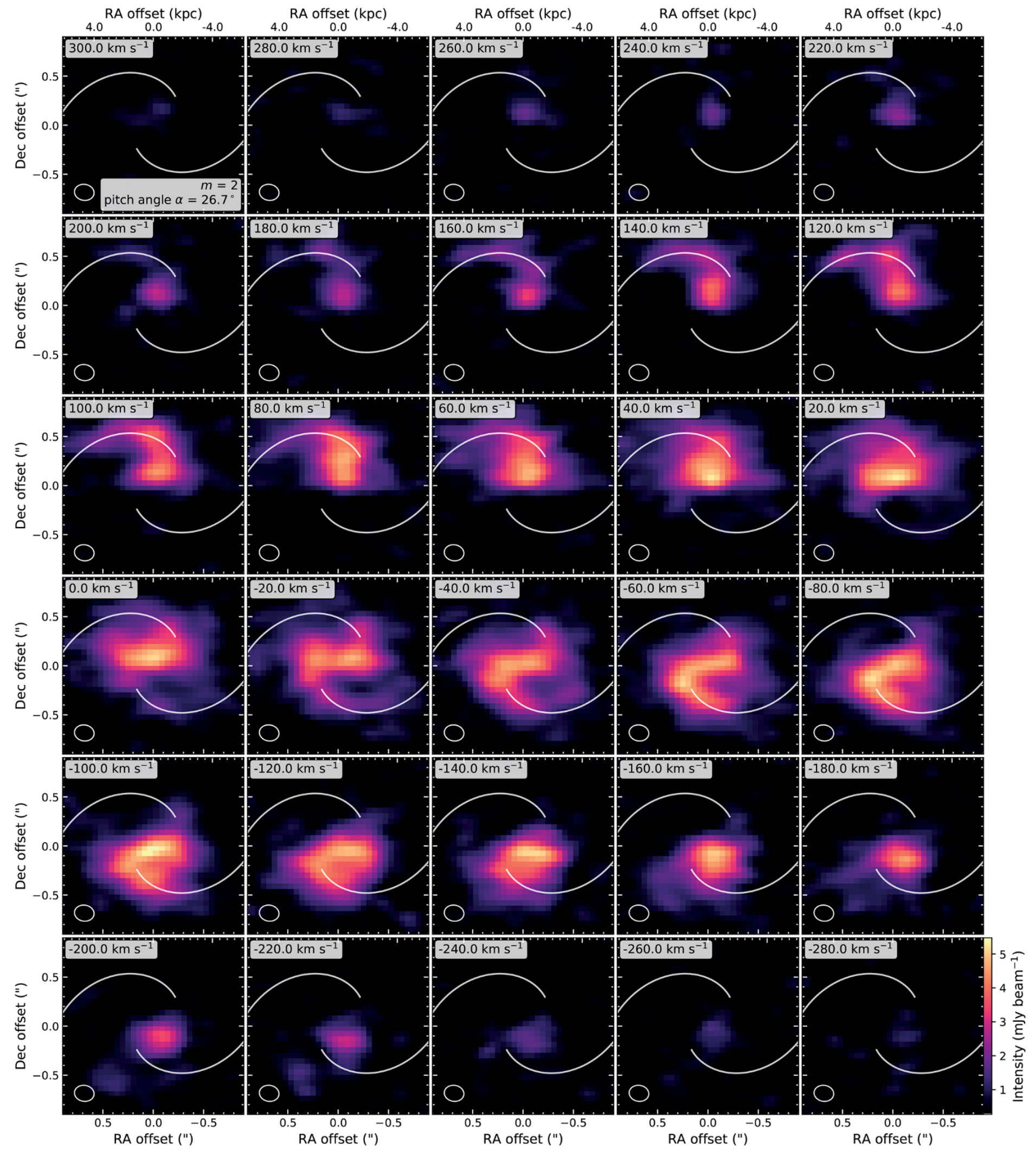


Fig. 2 (previous page). Velocity channel maps of the $[\mathrm{C} ॥]$ line emission, showing the spiral structure. Each panel shows an image at a specific velocity of [C II] line emission, labeled in the top left. Overlain with white lines is the best-fitting two-armed logarithmic spiral model, which has a pitch angle $\alpha=26.7^{\circ}(14)$. The images have been de-projected to be viewed face-on, using a position angle of $4.5^{\circ}$ and inclination of $37.8^{\circ}$ (table S1) (14). The FWHM of the synthesized beam is shown with the white ellipse in the left bottom of each panel.
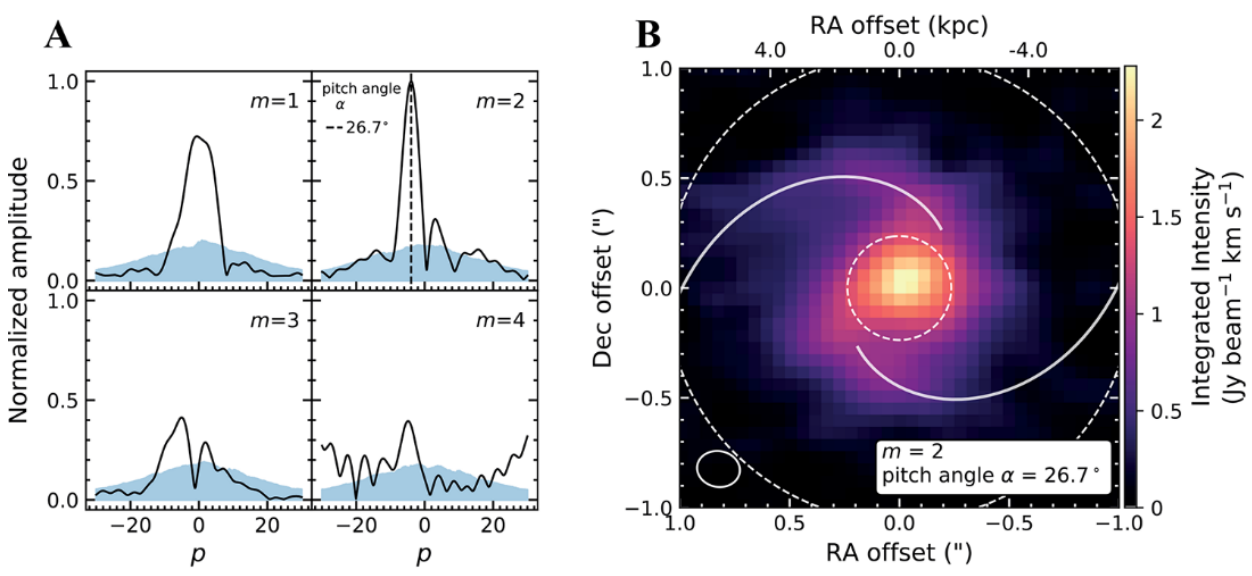

Fig. 3. Fourier analysis of the [C II] line emission. (A) Fourier spectra (14) of logarithmic spiral models with $m$ arms and pitch angles $\alpha$. The black solid lines show the normalized amplitude as a function of the dimensionless parameter $p=-m / \tan (\alpha)$. The blue shaded region indicates the estimated noise level (14). The $m=2$ mode has the strongest peak at a pitch angle of $26.7_{-1.6}^{+4.1 \circ}$ (dashed vertical line). (B) the [C II] line intensity image (deprojected version of Fig. $1 A$ ) overlaid with the best-fitting two-armed logarithmic spiral (white line, same as in Fig. 2). The FWHM of the synthesized beam is shown by an ellipse in the left bottom corner. The inner and outer dotted lines indicate the boundaries of the region used in the Fourier analysis (14). 